M. B. Christensen - D. A. Lawlor • T. R. Gaunt •

M.W. Howell · G. Davey Smith • S. Ebrahim •

I. N. M. Day

\title{
Genotype of galectin 2 (LGALS2) is associated with insulin-glucose profile in the British Women's Heart and Health Study
}

Received: 10 October 2005 / Accepted: 29 November 2005 / Published online: 9 February 2006

C) Springer-Verlag 2006

\begin{abstract}
Aims/hypothesis: It has been suggested that the gene encoding lymphotoxin-alpha (LTA) is associated with insulin resistance, and genetic association studies in the LTA region offer some support for this. However, LTA is in linkage disequilibrium with both the HLA gene cluster and the gene encoding TNF- $\alpha$, making inferences about causality difficult. In this study, we used the galectin 2 (LGALS2) genotype, which affects LTA secretion but is located on another chromosome than the HLA gene cluster or $T N F$, to examine the relationship between the LTA pathway and traits of the metabolic syndrome. Subjects: A cross-sectional genetic association study was carried out in 3,272 British women of European origin who were aged 60 to 79 years and were randomly selected from the community. Results: Fasting plasma glucose and serum insulin were statistically significantly associated with $L G A L S 2$ rs 7291467 , with this association being independent of BMI and WHR. The mean difference in fasting insulin per minor allele was $-4 \%(p=0.01$ for trend by allele) and the mean per minor allele difference in fasting glucose was $-2 \%$ ( $p=0.02$ for trend by allele). When women with known diabetes were excluded from the analyses the findings did not differ from those for the whole cohort. Conclusions/interpretation: Our findings for the physically unlinked $L G A L S 2$, invite further study of LGALS2 specifically and the LTA pathway generally for their influence on glucose-insulin regulation.
\end{abstract}

\footnotetext{
M. B. Christensen · T. R. Gaunt · M. W. Howell •

I. N. M. Day $(\bowtie)$

Human Genetics Division, School of Medicine,

Southampton University Hospital,

Duthie Building Mp808, Tremona Road,

Southampton, SO16 6YD, UK

e-mail: inmd@soton.ac.uk

Tel.: +44-23-80795063

Fax: $+44-23-80794264$
}

D. A. Lawlor · G. Davey Smith - S. Ebrahim - I. N. M. Day Department of Social Medicine, University of Bristol,

Bristol, UK
Keywords Genetic epidemiology · Glucose · Insulin • LGALS2 - Lymphotoxin- $\alpha$

Abbreviations dNTP: deoxynucleotide triphosphate . HDL-c: HDL cholesterol - LD: linkage disequilibrium LGALS2: galectin 2 - LTA: lymphotoxin-alpha $\cdot$ SNP: single nucleotide polymorphism $\cdot \mathrm{TNF}-\alpha$ : tumour necrosis factor-alpha

\section{Introduction}

Since the development of type 2 diabetes features insulin resistance, genes contributing to this trait remain candidates for type 2 diabetes. The inflammatory cytokines lymphotoxin- $\alpha$ (LTA) and TNF- $\alpha$ play multiple roles in the development and function of the immune system and several studies have reported associations between these cytokines and insulin resistance [1]. In support of a role for LTA in the pathogenesis of metabolic traits, common genotypes of LTA have been shown to be associated with traits of the metabolic syndrome, type 2 diabetes and myocardial infarction [2-5] However, LTA and TNF- $\alpha$ are homologous, and their genes are in significant linkage disequilibrium (LD) [6]. $L T A$ and $T N F$ are also in LD with the extensive $H L A$ cluster. Thus it is difficult to make causal inferences about LTA from assessing the association of its gene with pathological outcomes, as any association could in fact represent an effect of LTA, TNF- $\alpha$ or products from the $H L A$ cluster.

A recent search for factors interacting with LTA, identified galectin-2 (LGALS2) as co-localising with and binding to LTA [5] and a recent case control study in a Japanese population found an association between a common polymorphism (SNP rs7291467) of LGALS2 and myocardial infarction [5]. Functional studies have indicated that LTA +252 and LGALS2 rs7291467 can influence LTA levels $[4,5]$ and the authors of the Japanese study hypothesised that the effect of the LGALS2 polymorphism on myocardial infarction was likely to be via its effect on the secretion of LTA [5]. Since LGALS2 is located 
on chromosome 22q it is unlinked with the $H L A$ cluster or $T N F$ on chromosome 6 . Thus, LGALS2 offers the possibility to test the association between LTA secretion and traits of the metabolic syndrome, unconfounded by the syntenies and linkage disequilibria inherent in the $H L A$ region. An association of this polymorphism with metabolic syndrome traits would imply a discrete effect of LTA that is not due to its strong interactions with proteins, such as TNF- $\alpha$, with which it is related. It should also be noted that several family-based studies have identified genetic linkage of some metabolic syndrome traits to $22 \mathrm{q}$. The HyperGEN Network found linkage of diabetes status to 22q12.1 in hypertensive patients [7]; markerD22S683 near CYP2D6 on 22q was found to be linked and associated with type 2 diabetes in Northern Ontario Oji-Cree [8]; and the plasma HDL-cholesterol (HDL-c) level was linked to $22 \mathrm{q}$ in the Hong Kong Family Diabetes Study [9]. LGALS2 is located at 22q13.1 [5]: the respective linkages peak nearby at flanking locations, $22 \mathrm{q} 12.1$ and $22 \mathrm{q} 13.2$. In this study, we examined LGALS2 SNP rs7291467 in relation to metabolic syndrome traits in the British Women's Heart and Health Study.

\section{Subjects and methods}

Study and study participants

Data from the British Women's Heart and Health Study were used. Full details of this study have been previously reported [10]. Of the 4,278 participants who gave consent for genetic testing, 15 (five Afro-Caribbean, eight South Asian and two other) were defined by the examining nurse as being not of European origin and were excluded from further analysis. Of the remaining 4,263 women, 3,817 $(89 \%)$ had successful blood samples (for the remainder it was either impossible to obtain sufficient blood or the woman refused blood sampling) and 3,553 (83\%) had DNA available for genotyping. Fasting blood samples were used to measure serum insulin, plasma glucose, triglyceride and HDL, using standard procedures. Resting blood pressure (mean of two examinations), height, weight, waist and hip circumference were all assessed using standard procedures. Serum insulin was measured using an ELISA assay which does not cross react with proinsulin [11].

\section{Genotyping}

DNA extraction, quantitation and pre-amplification were conducted as described previously [10]. LGALS2 SNP rs7291467 was determined by LightTyper methodology (Roche Diagnostics, Mannheim, Germany) [12] using probes 5'-ACAGACACTCACAGACGTGTGCCCTGdabcyl-3'and and 5'-fluorescein-CACACACACGTCTAAC ACCA-Phosphate-3'. PCR primers were LGALS2 reverse: 5'-AGCCTGGGCGACAGAGCGAGAC-3' and LGALS2 forward: 5'-TGTTGCAGGGCTCGGGGTGT-3'. PCR conditions were: $1 \times$ Taq buffer (Promega, Southampton, UK) $0.2 \mathrm{mmol} / \mathrm{l} \mathrm{dNTPs}$ (Promega), $1.5 \mathrm{mmol} / \mathrm{l} \mathrm{MgCl} 2$ (Promega), right primer $0.5 \mu \mathrm{mol} / 1$, left primer $0.1 \mu \mathrm{mol} / 1$, fluorescein-probe $0.1 \mu \mathrm{mol} / 1$, dabcyl-probe $0.2 \mu \mathrm{mol} / 1$, Taq $0.01 \mathrm{U} / \mu \mathrm{l} \mathrm{PCR}$ (Promega), cycling $94^{\circ} \mathrm{C} 2 \mathrm{~min}, 94^{\circ} \mathrm{C} 20 \mathrm{~s}$, $68^{\circ} \mathrm{C} 30 \mathrm{~s}, 72^{\circ} \mathrm{C} 1 \mathrm{~min}$, step 2 to 449 times, $72^{\circ} \mathrm{C} 2 \mathrm{~min}$. Real time fluorescence change was then measured in a 384well plate LightTyper instrument (Roche Diagnostics, Cat. No. 03357414001). Genotypes were automatically identified in software, followed by manual validation. The success rate was $92 \%$, manual validation of all genotypes ensures good accuracy and assay patterns fell into three distinct groups. Since alleles are randomly allocated at gametogenesis we would not expect genotype to be associated with the socioeconomic and lifestyle factors that are related to traits of the metabolic syndrome and would therefore not expect the genotype-phenotype associations examined here to be confounded [13]. However, to establish that there was indeed no association, we examined the distributions of socioeconomic position (adult and childhood occupational social class), smoking and physical inactivity by genotype. Information on these characteristics were obtained from the baseline study questionnaire and the research nurse interview as previously described [14].

\section{Statistical analysis}

Means are presented by genotype. SNP rs7291467 is thought to affect transcription and in consequence, levels of galectin-2 and other proteins such as LTA. The effect is moderate and both alleles are expected to contribute to the phenotype. An additive genetic model was therefore considered most appropriate for analyses, consistent with the genetics of many quantitative traits. An $F$-test (one degree of freedom) was computed to assess the per allele effect. Linear regression was used to determine the mean difference per allele. Stratified analyses (using thirds of BMI and WHR) and $F$-tests were used to assess interactions between measures of adiposity (BMI and WHR) and metabolic outcomes (insulin, glucose, triglycerides and HDL-c). Insulin, glucose and triglycerides were positively skewed; geometric means are shown and the natural logs of the values were used in the regression models. The resultant regression coefficients were back transformed to give a ratio of geometric means from which a proportionate (\%) difference per allele was calculated. For the main analysis all women with genotype and phenotype data were included. Because women who had been diagnosed with type 2 diabetes would be receiving treatment, their fasting blood glucose levels are unlikely to represent their offtreatment levels. Therefore we repeated the analyses for genotype associations with fasting glucose and insulin excluding the 165 (5\%) women who had a clinical diagnosis of type 2 diabetes and were on treatment (any of diet and lifestyle only, oral hypoglycaemic agents or insulin). All analyses were conducted using Stata version 8 . 
Study ethics

Local ethics committee approvals were obtained for the British Women's Heart and Health Study. Participants were asked for informed consent to review their medical records and for permission to perform anonymised genetic tests on stored blood. Eight women declined to give consent and were excluded from this study.

\section{Results}

Of those subjects for whom DNA samples were available, $3,272(92 \%)$ had genotypic data. There was no difference in age, BMI or WHR between those with and without genotypic data (all $p$ values $>0.5$ ). Of the 3,272 who were genotyped $1,210(37.0 \%)$ were homozygous for the major allele, $1527(46.7 \%)$ were heterozygous and 535 (16.4\%) were homozygous for the minor allele. The genotype frequencies were in Hardy-Weinberg equilibrium $(p=0.15)$.

LGALS2 rs7291467 was associated with fasting insulin and glucose, such that levels of both were lower with the recessive allele (Table 1). Mean triglyceride levels, HDL-c, BMI, WHR and systolic blood pressure did not differ by genotype (Table 1). As anticipated, there were no associations between genotype and socioeconomic position or lifestyle risk factors and hence these could not confound any association between genotype and glucose or insulin (Table 2). This was further confirmed by adding these variables in multivariable analyses and noting that there was no effect on any of the associations presented in Table 1. The associations between LGALS2 and fasting insulin and glucose were the same within strata of BMI and WHR (all $p$ values for interactions $>0.7$ ). Our results suggest some gradation of effect across the three genotype groups, both for glucose and insulin, suggestive of a quantitative per-allele (4\%) effect, without any dependence of effect on BMI or WHR. The direction of effect is toward lower glucose and insulin for the minor allele $(\mathrm{C}: \mathrm{G}$ base pair). We note that the insulin effect was mainly attributable to a higher insulin level in T homozygotes.
Of the 3,272 women, $165(5 \%)$ had been clinically diagnosed with type 2 diabetes and were receiving some form of treatment (dietary and lifestyle advice and/or medication). When we repeated the analyses with these women excluded, the inverse per-allele association with fasting serum insulin remained unchanged to that for the whole cohort: $-4 \%(95 \% \mathrm{CI}:-6,-1 \%)$; the inverse association with fasting plasma glucose was weaker but still significant $(p=0.04):-1 \%(95 \% \mathrm{CI}:-2,0 \%)$. Though imprecise, the magnitude of effects presented here did not differ by area (South East England, Midlands or Wales, North England, Scotland) of birth for the women (all $p$ values for interactions of genotype with area of birth $>0.5$ ).

\section{Discussion}

Among women of European origin aged 60 to 79 years we found that lower fasting insulin and glucose levels were associated with LGALS2 rs7291467. LGALS2 rs7291467 was not associated with BMI, WHR, triglyceride levels, HDL-c or blood pressure. Previous studies have implicated LTA in coronary event pathogenesis and in metabolic syndrome traits and have also implicated LGALS2 in coronary event pathogenesis $[4,5,15]$. This study implicates $L G A L S 2$ in metabolic syndrome traits. As the study is cross-sectional, it may be prone to survivor bias. However, the lack of any association between genotype and age (Table 1) suggests that there is no clear survival advantage of any specific genotype. It is notable, considering the clear effect on fasting insulin and glucose, that there is no association with fasting triglyceride levels. The results presented here are not corrected for multiple testing. The metabolic syndrome traits are strongly correlated and it is therefore difficult to make a correction. If the metabolic syndrome is seen as having three aetiological categories (obesity-related, insulin resistancerelated and other), as has been proposed [16], a Bonferroni correction of three could be applied. The insulin results would still be statistically significant. We have chosen to genotype only SNP rs7291467. SNP rs7291467 was

Table 1 Association of $L G A L S 2$ with fasting serum insulin, plasma glucose and other components of the metabolic syndrome among British women aged 60 to 79 years $(n=3,272)$

\begin{tabular}{lccccc}
\hline & \multicolumn{3}{c}{ Mean (SD) } & Per-allele mean difference & $p$ value per allele (1df) \\
\cline { 2 - 4 } & TT $n=1,210$ & TC $n=1,527$ & CC $n=535$ & & 0.01 \\
\hline Insulin $(\mu \mathrm{unit} / \mathrm{l})^{\mathrm{a}}$ & $7.3(7.1,7.6)$ & $6.9(6.6,7.1)$ & $6.8(6.5,7.2)$ & $-4 \%(-7,-1 \%)$ & 0.02 \\
${\text { Glucose }(\mathrm{mmol} / \mathrm{l})^{\mathrm{a}}}_{\text {Triglycerides }(\mathrm{mmol} / \mathrm{l})^{\mathrm{a}}}$ & $6.0(5.9,6.1)$ & $5.9(5.9,6.0)$ & $5.8(5.7,5.9)$ & $-2 \%(-2,-1 \%)$ & 0.9 \\
HDL-c $(\mathrm{mmol} / \mathrm{l})$ & $1.67(1.63,1.72)$ & $1.70(1.66,1.74)$ & $1.66(1.60,1.73)$ & $0 \%(-2,2 \%)$ & 0.6 \\
BMI $\left(\mathrm{kg} / \mathrm{m}^{2}\right)$ & $27.6(5.0)$ & $27.6(5.1)$ & $27.5(4.9)$ & $-0.03(-0.28,0.21)$ & 0.8 \\
WHR $(\times 100)$ & $82.1(6.7)$ & $82.0(6.8)$ & $81.8(7.0)$ & $-0.18(-0.52,0.15)$ & 0.3 \\
Systolic BP $(\mathrm{mmHg})$ & $147.7(25.4)$ & $148.0(25.4)$ & $146.7(25.9)$ & $-0.33(-1.57,0.91)$ & 0.6 \\
Age (years) & $69.0(5.5)$ & $68.8(5.4)$ & $68.8(5.5)$ & $-0.13(-0.40,0.14)$ & 0.3 \\
\hline
\end{tabular}

${ }^{\mathrm{a}}$ Geometric mean and $95 \%$ CI of geometric mean 
Table 2 Association of $L G A L S 2$ with socioeconomic and lifestyle characteristics among British women aged 60 to 79 years $(n=3,272)$

\begin{tabular}{lllll}
\hline & $N(\%)$ & & $p$ \\
\cline { 2 - 4 } & $\mathrm{TT}$ & $\mathrm{TC}$ & $\mathrm{CC}$ & \\
& $n=1,210$ & $n=1,527$ & \multicolumn{1}{l}{$n=535$} & \\
\hline Current smoker & $135(11.2)$ & $176(11.5)$ & $68(12.7)$ & 0.6 \\
Physically inactive & $256(21.2)$ & $281(18.5)$ & $107(20.0)$ & 0.2 \\
Adult manual & $711(58.8)$ & $862(56.5)$ & $284(53.1)$ & 0.1 \\
social class & & & & \\
Childhood manual & $970(80.2)$ & $1217(79.7)$ & $427(79.8)$ & 0.8 \\
social class & & & & \\
\hline
\end{tabular}

$p$ value for difference across genotype categories for 2 degrees of freedom

identified in an association scan as having the strongest association with myocardial infarction and has in itself been shown to exert functional effects. Furthermore HapMap data show nearly perfect LD (all pairwise $r^{2}$ close to 1) for the haplotype block containing rs 7291467 . Therefore other SNPs were not examined, rs7291467 being both functional as well as a tagSNP $[17,18]$.

Ozaki et al. [5] observed, in a Japanese population, that the minor allele was the A:T base pair. The allele frequencies observed in our study are consistent with dbSNP data for subjects of European origin in whom the C: $\mathrm{G}$ base pair represents the minor allele, a population difference confirmed by the HapMap project [19]. In their implication of SNP rs7291467 in the pathogenesis of myocardial infarction, Ozaki et al. [5] found the A:T base pair to be protective in homozygotes. We found the A:T base pair to be associated with the highest level of insulin, particularly for homozygotes, and perhaps indicating some threshold effect. Our findings confirm the previously observed pattern where levels of LTA influence both insulin resistance and myocardial infarction in opposite directions $[4,15]$. These findings are contrary to predicted effects, if insulin resistance mediates the link between LTA and myocardial infarction. However, LTA could be exerting independent effects in the vascular wall and in the metabolic system, by acting through different members (TNFR1, TNFR2, HveA and LTBR) of the TNF- $\alpha$ receptor superfamily [6].

Our prior hypothesis was that there is a functional interaction between LTA and galectin-2 and, taken in conjunction with the independent genomic location of LGALS2, the examination of gene associations with LGALS2 is a valuable means to study the causal role of the LTA pathway in disease pathogenesis. Thus our results suggest LTA secretion plays a role in the development of insulin resistance.

In conclusion, we have found an association between LGALS2 and fasting insulin and glucose. This association supports the notion of a role for LTA in determining insulin-glucose profile. The $L G A L S 2$ genotype particularly and LTA pathway generally merit further exploration of their role in insulin metabolism.
Acknowledgements The British Women's Heart \& Health Study is co-directed by S. Ebrahim, D. A Lawlor, P. Whincup and G. Wannamethee. We thank C. Bedford, A. Emerton, N. Frecknall, K. Jones, R. Patel, M. Taylor and K. Wornell for collecting and entering data, all of the general practitioners and their staff who have supported data collection, and the women who have participated in the study. The British Women's Heart and Health Study is funded by the Department of Health and British Heart Foundation. D.A. Lawlor is funded by a UK Department of Health Career Scientist Award. Work in the laboratory of I. M. Day is funded by the British Heart Foundation and by the UK Medical Research Council. T.R. Gaunt is a British Heart Foundation Intermediate Fellow.

\section{References}

1. NCBI (2005) Genetic association database. http:// geneticassociationdb.nih.gov, accessed 10 April 2005

2. Yamada A, Ichihara S, Murase Y et al (2004) Lack of association of polymorphisms of the lymphotoxin alpha gene with myocardial infarction in Japanese. J Mol Med 82:477-483

3. Hamid YH, Urhammer SA, Glumer C et al (2005) The common T60N polymorphism of the lymphotoxin-alpha gene is associated with type 2 diabetes and other phenotypes of the metabolic syndrome. Diabetologia 48:445-451

4. Ozaki K, Ohnishi Y, Iida A et al (2002) Functional SNPs in the lymphotoxin-alpha gene that are associated with susceptibility to myocardial infarction. Nat Genet 32:650-654

5. Ozaki K, Inoue K, Sato H et al (2004) Functional variation in LGALS2 confers risk of myocardial infarction and regulates lymphotoxin-alpha secretion in vitro. Nature 429:72-75

6. Locksley RM, Killeen N, Lenardo MJ (2001) The TNF and TNF receptor superfamilies: integrating mammalian biology. Cell 104:487-501

7. Palmieri V, Bella JN, Arnett DK et al (2001) Effect of type 2 diabetes mellitus on left ventricular geometry and systolic function in hypertensive subjects: hypertension genetic epidemiology network (HyperGEN) study. Circulation 103:102-107

8. Hegele RA, Kwan K, Harris SB, Hanley AJ, Zinman B, Cao H (2000) NAT2 polymorphism associated with plasma glucose concentration in Canadian Oji-Cree. Pharmacogenetics 10:233238

9. Ng MCY, So W-Y, Lam VKL et al (2004) Genome-wide scan for metabolic syndrome and related quantitative traits in Hong Kong Chinese and confirmation of a susceptibility locus on chromosome 1q21-q25. Diabetes 53:2676-2683

10. Lawlor DA, Day IN, Gaunt TR et al (2004) The association of the PON1 Q192R polymorphism with coronary heart disease: findings from the British Women's Heart and Health cohort study and a meta-analysis. BMC Genet 5:17

11. Andersen L, Dinesen B, Jorgensen PN, Poulsen F, Roder ME (1993) Enzyme immunoassay for intact human insulin in serum or plasma. Clin Chem 39:578-582

12. Gaunt TR, Hinks LJ, Christensen MB, Kiessling M, Day IN (2005) Experience applying lightTyper methodology to human SNP's relevant to growth and cardiovascular risk. In: Hecker K (ed) Genetic variance detection: technologies for pharmacogenomics. DNA Press

13. Davey Smith G, Ebrahim S (2003) 'Mendelian randomization': can genetic epidemiology contribute to understanding environmental determinants of disease? Int J Epidemiol 32:1-22

14. Lawlor DA, Ebrahim S, Davey Smith G (2004) The metabolic syndrome and coronary heart disease in older women: findings from the British Women's Heart and Health Study. Diabet Med 21:906-913

15. Hayakawa T, Nagai Y, Taniguchi M et al (2000) Tumor necrosis factor-[beta] gene Nco I polymorphism decreases insulin resistance in Japanese men. Metab Clin Exp 49:15061509 
16. Grundy SM, Brewer HB Jr, Cleeman JI, Smith SC Jr, Lenfant C (2004) Definition of metabolic syndrome: report of the National Heart, Lung, and Blood Institute/American Heart Association conference on scientific issues related to definition. Circulation 109:433-438

17. Gabriel SB, Schaffner SF, Nguyen H et al (2002) The structure of haplotype blocks in the human genome. Science 296:22252229
18. Carlson CS, Eberle MA, Rieder MJ, Yi Q, Kruglyak L, Nickerson DA (2004) Selecting a maximally informative set of single-nucleotide polymorphisms for association analyses using linkage disequilibrium. Am J Hum Genet 74:106-120

19. HapMap (2005) The HapMap project. http://www.hapmap.org, accessed 10 July 2005 